\title{
Advances in schizophrenia research: first special issue, 2020
}

\author{
Julio Licinio ${ }^{1}$ \\ (c) Springer Nature Limited 2020
}

We are delighted to present our readers with a series of special issues that will be published in 2020 and beyond. This is the first of those issues, with a focus on schizophrenia. I will introduce here the outstanding papers on this major psychiatric disorder of immense public health relevance.

The paper by Passos and Mwangi covers the increasingly crucial approach of using machine learning to predict treatment response at the individual level [1]. Treatment prediction is also addressed by Cao et al., using brain functional connectivity [2]. Moreover, baseline brain structural and functional predictors of clinical outcome in the early course of schizophrenia are identified by Doucet et al. [3].

Scott-Price et al. demonstrate that genetic liability to schizophrenia is negatively associated with educational attainment in the UK Biobank [4]. Key data from the UK Biobank are also used in a study by Warland et al. on schizophrenia-associated genomic copy number variants and subcortical brain volumes. Those authors show that $\mathrm{CNV}$ carriers have reduced volume in some of the subcortical structures previously shown to be reduced in schizophrenia and that these associations partially accounted for the association between pathogenic copy number variants and cognitive impairment, a cardinal feature of schizophrenia [5]. Smeland et al. conducted a genome-wide analysis that revealed extensive genetic overlap between schizophrenia, bipolar disorder, and intelligence. Their study demonstrates that schizophrenia and bipolar disorder share genetic influences with intelligence, albeit in a different manner, providing new insights into their genetic architectures [6]. Comparisons of schizophrenia to other psychiatric disorders is also carried out by Koshiyama et al. [7]. They compared white matter microstructural differences

Julio Licinio

juliolicinio@gmail.com

1 State University of New York, Upstate Medical University, Syracuse, NY 13210, USA between healthy comparison subjects and patients with schizophrenia, bipolar disorder, autism spectrum disorder, or major depressive disorder. In comparison with controls, they found that schizophrenia, bipolar disorder, and autism spectrum disorder share similar white matter microstructural differences in the body of the corpus callosum; schizophrenia and bipolar disorder featured comparable changes in the limbic system, such as the fornix and cingulum. Alterations in tracts connecting neocortical areas, such as the uncinate fasciculus, were observed only in schizophrenia, with no significant difference found in depression. Significant differences between schizophrenia/bipolar disorder and major depressive disorder were found in the limbic system.

In this issue we have articles examining the role of transmitters long thought to be involved the pathophysiology of schizophrenia as well as articles that point out new directions. In terms of existing candidates, the article by Petrelli et al. looks at dysfunction of homeostatic control of dopamine by astrocytes in the developing prefrontal cortex and how that leads to cognitive impairments [8]. The roles of glutamate, glutamate receptors, and glutathione are explored in papers by Kumar et al. and Wang et al., respectively $[9,10]$. New approaches are presented in several articles. Cai et al. show increased macrophages and changed brain endothelial cell gene expression in the frontal cortex of people with schizophrenia displaying inflammation [11]. In the same broad area of brain immunity, Dwir et al. demonstrate that MMP9/RAGE pathway overactivation mediates redox dysregulation and neuroinflammation, leading to inhibitory/excitatory imbalance [12]. Proteasome dysfunction in postmortem cortex in schizophrenia was examined by Scott and Meador-Woodruff [13]. Reay and colleagues have two interesting articles that shed new light on the role of retinoids in schizophrenia [14, 15].

Genetic aspects of schizophrenia are covered in several papers in this thematic issue that examine not only genetic data, but also related functional alteration. That critical topic is covered by papers by Radulescu et al. [16], Bergman et al. [17], Chaumette et al. [18], and Ma et al. [19]. 
A new therapeutic approach is provided by Hadar et al., who show that application of noninvasive transcranial direct current stimulation during adolescence, prior to schizophrenia-relevant behavioral manifestation, prevents the development of positive symptoms and related neurobiological alterations in the maternal immune stimulation model of schizophrenia [20].

These outstanding articles contribute to expand the frontiers of existing knowledge in schizophrenia, covering the full range from molecules, transmitters, and genes to new insights into the underlying biology, imaging correlates, and outcomes as well as pointing out a new direction toward a novel treatment approach.

Publisher's note Springer Nature remains neutral with regard to jurisdictional claims in published maps and institutional affiliations.

\section{References}

1. Passos IC, Mwangi B. Machine learning-guided intervention trials to predict treatment response at an individual patient level: an important second step following randomized clinical trials. Mol Psychiatry. 2018. https://doi.org/10.1038/s41380-018-0250-y.

2. Cao B, et al. Treatment response prediction and individualized identification of first-episode drug-naive schizophrenia using brain functional connectivity. Mol Psychiatry. 2018. https://doi.org/10. 1038/s41380-018-0106-5.

3. Doucet GE, Moser DA, Luber MJ, Leibu E, Frangou S. Baseline brain structural and functional predictors of clinical outcome in the early course of schizophrenia. Mol Psychiatry. 2018. https://doi. org/10.1038/s41380-018-0269-0.

4. Escott-Price V, et al. Genetic liability to schizophrenia is negatively associated with educational attainment in UK Biobank. Mol Psychiatry. 2019. https://doi.org/10.1038/s41380-018-0328-6.

5. Warland A, Kendall KM, Rees E, Kirov G, Caseras X. Schizophrenia-associated genomic copy number variants and subcortical brain volumes in the UK Biobank. Mol Psychiatry. 2019. https://doi.org/10.1038/s41380-019-0355-y.

6. Smeland $\mathrm{OB}$, et al. Genome-wide analysis reveals extensive genetic overlap between schizophrenia, bipolar disorder, and intelligence. Mol Psychiatry. 2019. https://doi.org/10.1038/s41380-018-0332-x.

7. Koshiyama D, et al. White matter microstructural alterations across four major psychiatric disorders: mega-analysis study in
2937 individuals. Mol Psychiatry. 2019. https://doi.org/10.1038/ s41380-019-0553-7.

8. Petrelli F, et al. Dysfunction of homeostatic control of dopamine by astrocytes in the developing prefrontal cortex leads to cognitive impairments. Mol Psychiatry. 2018. https://doi.org/10.1038/ s41380-018-0226-y.

9. Kumar J, et al. Glutathione and glutamate in schizophrenia: a 7T MRS study. Mol Psychiatry. 2018. https://doi.org/10.1038/ s41380-018-0104-7.

10. Wang HY, et al. mGluR5 hypofunction is integral to glutamatergic dysregulation in schizophrenia. Mol Psychiatry. 2018. https://doi.org/10.1038/s41380-018-0234-y.

11. Cai HQ, et al. Increased macrophages and changed brain endothelial cell gene expression in the frontal cortex of people with schizophrenia displaying inflammation. Mol Psychiatry. 2018. https://doi.org/10.1038/s41380-018-0235-x.

12. Dwir D, et al. MMP9/RAGE pathway overactivation mediates redox dysregulation and neuroinflammation, leading to inhibitory/ excitatory imbalance: a reverse translation study in schizophrenia patients. Mol Psychiatry. 2019. https://doi.org/10.1038/s41380019-0393-5.

13. Scott MR, Meador-Woodruff JH. Intracellular compartmentspecific proteasome dysfunction in postmortem cortex in schizophrenia subjects. Mol Psychiatry. 2019. https://doi.org/10.1038/ s41380-019-0359-7.

14. Reay WR, Cairns MJ. The role of the retinoids in schizophrenia: genomic and clinical perspectives. Mol Psychiatry. 2019. https:// doi.org/10.1038/s41380-019-0566-2.

15. Reay WR, et al. Polygenic disruption of retinoid signalling in schizophrenia and a severe cognitive deficit subtype. Mol Psychiatry. 2018. https://doi.org/10.1038/s41380-018-0305-0.

16. Radulescu E, et al. Identification and prioritization of gene sets associated with schizophrenia risk by co-expression network analysis in human brain. Mol Psychiatry. 2018. https://doi.org/10. 1038/s41380-018-0304-1.

17. Bergman O, Karry R, Milhem J, Ben-Shachar D. NDUFV2 pseudogene (NDUFV2P1) contributes to mitochondrial complex I deficits in schizophrenia. Mol Psychiatry. 2018. https://doi.org/10. 1038/s41380-018-0309-9.

18. Chaumette B, et al. Missense variants in ATP1A3 and FXYD gene family are associated with childhood-onset schizophrenia. Mol Psychiatry. 2018. https://doi.org/10.1038/s41380-018-0103-8.

19. Ma L, et al. Schizophrenia risk variants influence multiple classes of transcripts of sorting nexin 19 (SNX19). Mol Psychiatry. 2019. https://doi.org/10.1038/s41380-018-0293-0.

20. Hadar R, et al. Prevention of schizophrenia deficits via noninvasive adolescent frontal cortex stimulation in rats. Mol Psychiatry. 2019. https://doi.org/10.1038/s41380-019-0356-x. 